\title{
Day-Ahead Market Bidding for a Nordic Hydropower Producer: Taking the Elbas Market into Account
}

\author{
Eduardo Faria and Stein-Erik Fleten \\ Eduardo Faria \\ Department of Electrical Engineering, \\ Pontifical Catholic University of Rio de Janeiro (PUC-Rio), \\ Rua Marquês de São Vicente 225, Edificio Cardeal, Leme, sala 401(Secretaria), \\ Gávea - Rio de Janeiro - RJ, Cep: 22451-900, Brazil \\ Phone: +552181113393 \\ email: edufaria@ele.puc-rio.br
}

\author{
Stein-Erik Fleten \\ Department of Industrial Economics and Technology Management \\ Norwegian University of Science and Technology, NO-7491 Trondheim, Norway \\ phone: +4773591296 \\ email: stein-erik.fleten@iot.ntnu.no \\ url: http://www.iot.ntnu.no/users/fleten/
}

\begin{abstract}
In many power markets around the world the energy generation decisions result from two-sided auctions in which producing and consuming agents submit their price-quantity bids. The determination of optimal bids in power markets is a complicated task that has to be undertaken every day. In the present work, we propose an optimization model for a price-taker hydropower producer in Nord Pool that takes into account the uncertainty in market prices and both production and physical trading aspects. The day-ahead bidding takes place a day before the actual operation and energy delivery. After this round of bidding, but before actual operation, some adjustments in the dispatched power (accepted bids) have to be done, due to uncertainty in prices, inflow and load. Such adjustments can be done in the Elbas market, which allows for trading physical electricity up to one hour before the operation hour. This paper uses stochastic programming to determine the optimal bidding strategy and the impact of the possibility to participate in the Elbas. ARMAX and GARCH techniques are used to generate realistic market price scenarios taking into account both day-ahead price and Elbas price uncertainty. The results show that considering Elbas when bidding in the day-ahead market does not significantly impact neither the profit nor the recommended bids of a typical hydro producer.
\end{abstract}

\section{Keywords}

Stochastic programming, Mixed integer programming, Electricity auctions, Elbas,, Hydroelectric scheduling, GARCH 


\section{INTRODUCTION}

In recent years, many countries have deregulated their power sectors, leading to new challenges for energy producers and increasing the interest in power optimization approaches to develop appropriate bidding strategies. The liberalization of the electricity markets in the Nordic countries started in the beginning of the 1990s, with Norway as the pioneer. Already in 1993 the Nord Pool was established as a Norwegian electricity market, and extended its trade to Sweden in 1996, followed by Finland and Denmark in 1998 and 2000, respectively. It thus became the world's first multinational exchange for trade in electric power.

The Nordic power market has successfully adapted to the competitive environment and has inspired the restructuring of other power markets. The dayahead market in Nord Pool, the Elspot (also known as Nord Pool Spot), takes the form of a pool-based market in which market participants exchange power contracts for physical delivery the following operation day. The value of turnover traded in the Elspot in 2007 was EUR 9.1 billion and the energy volumes amounted to $291 \mathrm{TWh}$. This is more than $65 \%$ of the total electricity consumption in the Nordic countries, while the remaining is traded through bilateral contracts. Hence, the power sales in the day-ahead market constitute a substantial part of the revenues for the producers. This makes the bidding into the day-ahead market one of the most important tasks the power producers are faced with.

Like in many other power markets around the world, the dispatch in the Elspot is the result of two-sided auctions in which producing and consuming agents submit their price-quantity bids. Since day-ahead market clearing prices are determined by the balance between sales and purchase bids, the volumes dispatched and the prices at which transactions are settled are unknown until the market has been cleared and market clearing prices have been determined. In practice, short-term power planning and operation is often based on deterministic optimization tools, such as the Short-term Hydro Operation Planning (SHOP) model (Flatabø et al. 2002, Fosso and Belsnes 2004 and Fosso et al. 1999), widely used in Norway, whereas the bidding tasks rests on skills and experience of the operating engineers.

Bidding strategies are extremely relevant to hydropower producers, since they can store water in their reservoirs and use this flexibility to decide the best time to produce energy, according to the development in market prices. When prices are high, water is released and energy is produced and sold, whereas when prices are low, the water is saved for future use at higher prices. Therefore, the price uncertainty is an important aspect to be considered when developing a bidding strategy.

Regarding the literature on bidding strategies for hydropower generators, we give a few references. Fleten and Kristoffersen (2007) consider a Nordic hydropower producer that owns a series of power plants along a river and introduce a stochastic programming model to maximize the profit of its bidding strategy. The balancing market adjustments in generation are represented in a simplified way. Ladurantaye et al. (2007) also integrates bids and reserve sales in a deregulated electricity market using a stochastic programming model. Fleten and Pettersen 
(2005) consider a price-taker power marketer who supplies power to end users through purchases in the day-ahead market and propose a stochastic programming formulation for its bidding problem. The bidding is subject to market price uncertainty, which is represented by scenarios generated by moment matching (Høyland et al. 2003). Regarding strategies for thermal generators, Conejo et al. (2002) takes the perspective of a price-taker thermal power producer and develops a simple bidding strategy to bid in the day-ahead market. The expected value problem is solved, i.e., the stochastic prices are replaced by expected prices, and the optimal solution is used to build the bidding curves. Problems that comprise thermal and hydro generation are studied by Oliveira et al. (2003) and Shawwash et al. (2000), which develop deterministic short-term optimization models, whereas Nowak et al. (2003) present a stochastic version of the like. Philpott et al. (2000) represent uncertainty in demand and hydropower unit commitment in a multi-stage stochastic programming model. Finally, Anderson and Philpott (2000) study strategies of generators bidding in competitive power markets where the behaviors of other agents are uncertain and modeled by a so-called market distribution function. The bidding curves are determined by solving an optimal control problem. For power optimization models in stochastic programming similar to the present work, see the reviews of Wallace and Fleten (2003) and Kristoffersen and Fleten (2009), the last one with emphasis on short-term power production and trading problems.

After the spot prices are published (up to 14:00) and the accepted bids are set, the generators must send their production plans for the next day to the transmission system operators (TSO), before 19:00 every day. Possible imbalances between load and generation during the day are handled by the TSO within each country, who buy system services such as ramping and frequency control from the producers through short-term auctions.

Imbalances between volumes produced and volumes dispatched in the day-ahead market are settled in the regulating market, where the participants submit their bids to increase or decrease the generation (or consumption). Bids placed on the regulating market must be executable within a short period of time (15 minutes). For further details, see Olsson and Söder (2008), who model balancing market prices using combined seasonal auto regressive integrated moving average (SARIMA) and discrete Markov processes in order to generate scenarios considering the fact that in some periods there is no demand for balancing power.

Adjustments in the actual generation can also be done by trading energy in the Elbas market, which allows for trading physical electricity between the participants. Especially when the agents are allowed to trade energy in the Elbas market, the volume traded in the balancing market is very low, and therefore it is not taken into account in this work.

The objective of this work is to develop a model to support the day-ahead bidding of a price-taker producer taking into account the possibility of trading energy in the Elbas market.

The decision process is divided into stages. The day-ahead bids must be sent to Nord Pool before 12:00 every day, which means that there is a substantial gap between bidding and the actual generation. To a small market participant, whose decisions have no impact on the spot price, the bidding process is subject to uncertainty in day-ahead and Elbas prices. The problem is approximated as a two- 
stage mixed integer stochastic program. The first stage concerns day-ahead market exchange whereas the second stage takes in trades in the Elbas market and real-time hydropower production.

To a price-taker producer, i.e., that is sufficiently small and does not have market power to affect the prices with its bids, the market prices can be modeled as exogenous. The uncertainty in day-ahead prices and Elbas prices are represented by a set of scenarios generated by stochastic processes calibrated from historical data. The model we use is an ARMA with GARCH (Generalized Autoregressive Conditional Heteroskedastic) error components. The GARCH model, firstly introduced by which is already being used to analyze electricity time series (Garcia and Contreras 2005). The Elbas prices, which are very correlated with the day-ahead prices, are also modeled by an ARMAX with GARCH methodology, using day-ahead prices as explanatory variables.

The problem is solved as a deterministic equivalent (see Wets 1974, and Thénié et al. 2007), where the objective function is to maximize the expected value of the profit considering all the possible Elspot and Elbas prices scenarios. The model is implemented and solved with the commercial software Xpress-MP, providing, most importantly, the first stage solution, i.e., the bidding decisions into the dayahead market. The second stage decisions are the production schedule and trades in the Elbas market. Start-up and stop costs are modeled using binary variables. The value of considering the trades in the Elbas market is analyzed, and tests are done with data from three hydroelectric stations of Agder Energi, a medium sized power producer in Norway.

The outline of the present paper is as follows. We explain the composition of the day-ahead market and model the bidding process in Section 2. In Section 3 we describe the Elbas market. In Section 4 we model the short-term hydropower production. In Section 5 we introduce the price uncertainty and incorporate it into the stochastic programming model, whereas Section 6 is devoted to scenario generation. Section 7 illustrates a case study from a Norwegian hydropower producer and Section 8 presents the conclusions.

\section{DAY-AHEAD BIDDING}

The Elspot contracts commit generators and buyers to deliver or receive power for a period of one hour or longer. Contract types include hourly bids, block bids and flexible hourly bids. Bids consist of price and volume pairs that together constitute the bidding curve. Elspot makes linear interpolation between consecutive price-volume pairs to construct the bidding curve. For each buyer or seller, the volume dispatched is determined by the point on the bidding curve that corresponds to the calculated market-clearing price. All transactions are settled at the market price. Block bids are lumped bids valid for at least two consecutive hours and contain only one price and volume and are accepted or rejected as a whole. Roughly, a mean price condition determines whether a block bid is accepted or rejected; the average market price over the relevant hours must be higher (lower) than the block bid price for a sales (purchase) bid for the bid to be accepted. So-called flexible hourly bids are omitted here as the bids are mainly used by large industrial consumers that are able to temporarily reduce their load. 
The Elspot market clearing price calculation is based on a mixed integer program that seeks to maximize the welfare gain (consumers' utility minus producers' cost), subject to physical constraints such as transmission capacities, area balances (accepted supply plus net import equals accepted demand) and maximal transmission ramp rates (the transmission between two given areas in two successive hours should not differ more than a maximum allowed amount). Besides the constraints mentioned above, the market price is calculated considering the influence of the various types of block bids on the price calculation of successive hours.

The Nordic grid is divided into fixed price areas. Sweden, Finland, East and West Denmark are one area each, whereas Norway can be divided into several areas, usually between 2 and 4 . This is determined by the TSO for months at a time, which will depend on the expected market equilibrium, transmission capacities and demand for inter-zonal transmission. If the contractual flow between two areas does not exceed the grid capacity, their market prices are equal. Otherwise, different prices are calculated ${ }^{1}$.

This section describes the bidding process and how the decisions are represented in the model developed in this work. We assume that the producer does not have any bilateral exchange contract (as this does not affect the bidding problem), but instead concentrates the entire production in the day-ahead and Elbas markets.

The model is designed for a time horizon corresponding to the 24 hours of the next day, which is divided into hourly time intervals and is denoted $\mathcal{T}=\{1, \ldots, 24\}$. Within the 24 hours the set of blocks $\mathcal{B}=\left\{b_{1}, \ldots, b_{B}\right\}$ is constructed. A block is a number of minimum two consecutive hours and the total number of such blocks within 24 hours is $B=276$. However, for computational reasons, we consider in this work a set of 6 blocks of 4 consecutive hours $(B=6)$ : $b_{1}=\{1, \ldots, 4\}, \quad b_{2}=\{5, \ldots, 8\}, \quad b_{3}=\{9, \ldots, 12\}, \quad b_{4}=\{13, \ldots, 16\}$, $b_{5}=\{17, \ldots, 20\}$ and $b_{6}=\{21, \ldots, 24\}$.

The bidding process consists of selecting both bid prices and bid volumes, which would lead to a product of variables. However, following Fleten and Pettersen (2005), the nonlinearities are avoided by fixing prices in advance, so that only volumes have to be selected. Let $\mathcal{I}=\{1, \ldots, I\}$ index the possible bid prices and denote these prices $p_{i}, i \in \mathcal{I}$ where $p_{i} \leq p_{i+1}$. The corresponding bid volumes are represented by the variables $x_{i t} \in \mathbb{R}_{+}, i \in \mathcal{I}, t \in \mathcal{T}$ for hourly bids and $x_{i b} \in \mathbb{R}_{+}, i \in \mathcal{I}, b \in \mathcal{B} \quad$ for block bids. The variables $y_{t} \in \mathbb{R}_{+}, t \in \mathcal{T}$ and $y_{b} \in \mathbb{R}_{+}, b \in \mathcal{B}$ are the volumes dispatched, for hourly bids and block bids respectively. The hourly market prices are denoted $\rho_{t}, t \in \mathcal{T}$ and average market prices for the blocks $\bar{\rho}_{b}, b \in \mathcal{B}$ where $\bar{\rho}_{b}=(1 /|b|) \sum_{t \in b} \rho_{t}$. Thus, the total sales revenues of the producer is:

$$
\sum_{t \in \mathcal{T}} \rho_{t} y_{t}+\sum_{b \in \mathcal{B}} \bar{\rho}_{b} y_{b}
$$

Hourly bids are handled as in Fleten and Kristoffersen (2007). For each hour $t \in \mathcal{T}$, the bids $\left(x_{i t}, p_{i}\right), i \in \mathcal{I}$ are interpreted as price-volume points on a bidding

\footnotetext{
${ }^{1}$ For further details visit the Nord Pool Spot AS website: http://www.nordpoolspot.com .
} 
curve that determines the relation between volumes bid and volumes dispatched. The curve is constructed by making a linear interpolation between the points which results in a piecewise linear curve. Thus, in terms of prices, the bidding curve can be expressed as (see Figure 1):

$$
\rho_{t}= \begin{cases}p_{1}+\frac{p_{2}-p_{1}}{x_{2 t}-x_{1 t}}\left(y_{t}-x_{1 t}\right) & \text { if } x_{1 t} \leq y_{t}<x_{2 t} \\ \vdots & \\ p_{i-1}+\frac{p_{i}-p_{i-1}}{x_{i t}-x_{i-1, t}}\left(y_{t}-x_{i-1, t}\right) & \text {,if } x_{i-1, t} \leq y_{t}<x_{i t} \\ \vdots & \\ p_{I-1}+\frac{p_{I t}-p_{I-1, t}}{x_{I t}-x_{I-1, t}}\left(y_{t}-x_{I-1, t}\right) & , \text { if } x_{I-1, t} \leq y_{t} \leq x_{I t}\end{cases}
$$

or equivalently, in terms of volumes:

$$
y_{t}= \begin{cases}\frac{\rho_{t}-p_{1}}{p_{2}-p_{1}} x_{2 t}+\frac{p_{2}-\rho_{t}}{p_{2}-p_{1}} x_{1 t} & \text {,if } p_{1} \leq \rho_{t}<p_{2} \\ \vdots & \\ \frac{\rho_{t}-p_{i-1}}{p_{i}-p_{i-1}} x_{i t}+\frac{p_{i}-\rho_{t}}{p_{i}-p_{i-1}} x_{i-1, t} & \text {,if } p_{i-1} \leq \rho_{t}<p_{i} \\ \vdots & \\ \frac{\rho_{t}-p_{I-1}}{p_{I}-p_{I-1}} x_{I t}+\frac{p_{I}-\rho_{t}}{p_{I}-p_{I-1}} x_{I-1, t} & \text {,if } p_{I-1} \leq \rho_{t} \leq p_{I} .\end{cases}
$$

Considering the sales bids from the perspective of a producer, it would be natural for the bidding curve to be increasing ${ }^{2}$ :

$$
x_{i t} \leq x_{i+1, t}, \quad i \in \mathcal{I} \backslash\{I\}, \quad t \in \mathcal{T}
$$

The block bids are $\left(x_{i b}, p_{i}\right), i \in \mathcal{I}$ and, for each $b \in \mathcal{B}$, the relation between volumes bid and volumes dispatched is

$$
y_{b}=\sum_{j: p_{j} \leq \bar{\rho}_{b}} x_{j b}, \quad b \in \mathcal{B}
$$

i.e., in a given block, the volume dispatched comprises the volumes of accepted bids. For example, consider the block $b_{2}=\{5, \ldots, 8\}$. If two bids are given by $\left(x_{1,2}, p_{1}\right)=(100,120)$ and $\left(x_{2,2}, p_{2}\right)=(200,150)$ and the average market price is $\bar{\rho}_{2}=130$, then only the first bid is accepted and the volume dispatched is $y_{2}=100$.

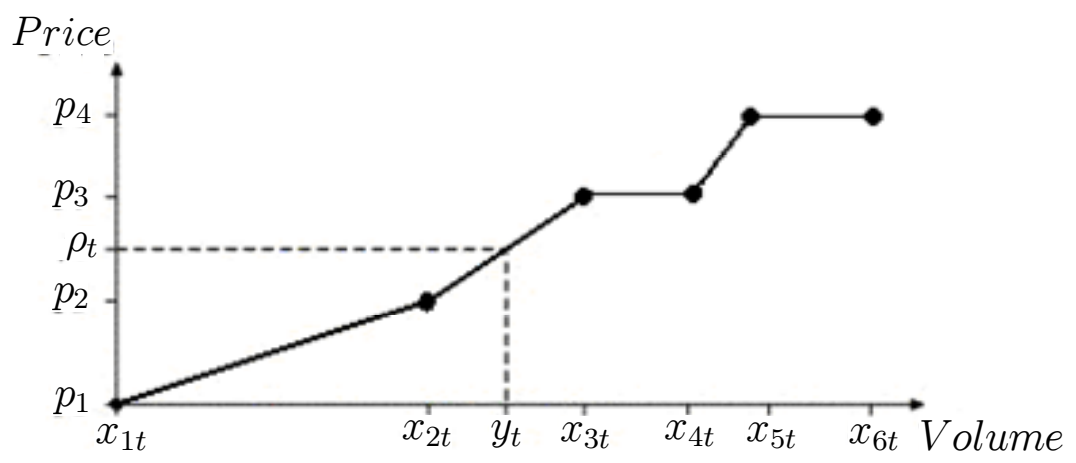

Figure 1 - Bidding curve of time interval $t \in \mathcal{T}$.

\footnotetext{
2 Nord Pool does not require that the bidding curve be increasing and therefore such constraints may in principle be omitted. Omitting the constraints, however, does not affect the optimal objective function values.
} 


\section{THE ELBAS MARKET ${ }^{3}$}

Adjustments in the actual generation (or consumption) during the day can be achieved by trading energy in the Elbas market, which allows the participants to start trading physical electricity for the next day at 14:00, when the spot prices for the next day are published. Trades are allowed up to one hour before the operation hour. Figure 2 shows the scheme of the day-ahead bidding and Elbas trading schedule.



Figure 2 - Schedule of the day-ahead bidding and Elbas trading

The Elbas market is open around the clock every day of the year and the traded products are $1 \mathrm{MWh}$ of electric power contracts, one for each specific hour. Today this market is open in Finland, Sweden, Denmark and Germany. Norway is joining the Elbas during the first quarter of 2009.

A bid in the Elbas consists of the bid type (sell or buy), a price in EUR/MWh and a volume in MWh for a specific hour. The trading process works as in a stock market, where the participants place their bids into a web based real-time trading system. The bids are anonymous and the trading system developed for continuous trading allows the participants to easily follow the situation on the market, place bids and search trade and cash-flow information.

Like in a stock market, there is always a gap between the minimum selling price and the maximum buying price. If one participant wants to sell energy immediately it must bid at a price at least as low as the maximum buying price, and if one wants to buy energy immediately, it must bid at a price at least as high as the minimum selling price. Considering the block bids in Elbas is left for future work.

The trading system automatically controls the cross-border capacity, which is given when the deadline for filing complaints on the Elspot has elapsed and the cross border capacity that is left after Elspot is known. For example if there is no transmission capacity from Finland to Sweden the participants in the Swedish and the Eastern Danish market area do not see the sale bids placed by participants in the Finnish market area in their trading system. If all the bids are inside the given

3 The source of information in this section is Nord Pool Spot AS website: http://www.nordpoolspot.com . 
cross border capacity the different market areas are treated as one.

To model the Elbas market we include the variable $z_{t} \in \mathbb{R}$, which represents the volume of energy traded in each hour $t \in \mathcal{T}$. The Elbas price for which the volume is traded is denoted $\varphi_{t}$. Since any participant can sell or buy energy on the Elbas market, the variable $z_{t}$ can be either positive, to represent a revenue when energy is sold, or negative, to represent a debit when energy is bought. Together with the results of the day-ahead market bidding defined in the previous section, the total sales revenues considering the trades in the Elbas market accumulate to:

$$
\sum_{t \in \mathcal{T}} \rho_{t} y_{t}+\sum_{b \in \mathcal{B}} \bar{\rho}_{b} y_{b}+\sum_{t \in \mathcal{T}} \varphi_{t} z_{t}
$$

The total energy traded in Elbas in 2007 amounted to $1.6 \mathrm{TWh}$; this is low compared to the Elspot trading (291 TWh in 2007) and to the regulating market (about 13 TWh in 2007). Norway, which is the second biggest country regarding total generation in Nord Pool, will make the total energy traded in Elbas increase. However, it is hard to predict how much larger this number will become.

Considering the relatively low liquidity on Elbas, and the fact that we are looking at a small market participant, we need to prevent the model's Elbas trading volume from becoming unrealistically high. Hence, we impose a limit on the volume of energy traded for each hour $t \in \mathcal{T}$. The model results are analyzed for 3 different limits, which are specified through percentages of the total installed capacity of the plants: $5 \%, 10 \%$ and $20 \%$. The results of the 3 cases are shown in Section 7.

\section{SHORT-TERM HYDROPOWER PRODUCTION}

This section introduces the part of the model related to the short-term hydropower production. Modeling is restricted to mixed-integer linear programming and follows the lines of Fleten and Kristoffersen (2008). The case study used in this work corresponds to a set of three hydropower plants from the upper part of the Otra cascade. Figure 3 shows the cascade with two plants (Holen12 and Holen3) located upstream of the third one (Brokke). The total capacity is $719 \mathrm{MW}$, divided among the 7 power units represented by the circles.



Figure 3 - Upper part of the Otra cascade used as case study

The hydropower production results from the coordinated operation of all three plants, and, in a simplified way, works as follows. Upstream water reaching each upper plant flows to the reservoirs where it is stored until released for generation. When released, the water flows to the next downstream reservoir, which is the 
same for both upstream stations. There, water is again stored for later generation. Electricity is generated by transforming the potential energy of the water into electricity. Water that is released but not discharged for generation is considered spill. After leaving each plant, the water proceeds downstream.

To model hydropower generation in a cascade, let $\mathcal{J}$ index the reservoirs and let $\mathcal{I}_{j}, j \in \mathcal{J}$, index the generators of the connected power stations. For the case study, $\mathcal{J}=\{1,2,3\}, \mathcal{I}_{1}=\{1,2\}, \mathcal{I}_{2}=\{3\}, \mathcal{I}_{3}=\{4,5,6,7\}$. Let the variables $u_{i t} \in\{0,1\}, i \in \mathcal{I}_{j}, j \in \mathcal{J}, t \in \mathcal{T}$ represent the on/off states of the generators, $w_{i t} \in \mathbb{R}^{+}, \quad i \in \mathcal{I}_{j}, j \in \mathcal{J}, t \in \mathcal{T} \quad$ the generation levels and $v_{i t} \in \mathbb{R}^{+}$, $i \in \mathcal{I}_{j}, j \in \mathcal{J}, t \in \mathcal{T}$ the corresponding discharges from each generator. Also, let the variables $l_{j t} \in \mathbb{R}^{+}, j \in \mathcal{J}, t \in \mathcal{T}$ be the reservoir storage levels and $r_{j t} \in \mathbb{R}^{+}$, $j \in \mathcal{J}, t \in \mathcal{T}$ the spill. As concerns direct costs of hydropower generation, operating costs are negligible. However, start-up costs amount to

$$
\sum_{t \in \mathcal{T}} \sum_{j \in \mathcal{J}} \sum_{i \in \mathcal{I}_{j}} S_{i}\left(u_{i, t-1}, u_{i t}\right)
$$

where the cost functions are

$$
S_{i}\left(u_{i, t-1}, u_{i t}\right)=c_{i} \max \left\{u_{i t}-u_{i, t-1}, 0\right\}, \quad i \in \mathcal{I}_{j}, \quad j \in \mathcal{J}, \quad t \in \mathcal{T}
$$

and the costs per start-up are $c_{i}, i \in \mathcal{I}_{j}, j \in \mathcal{J}$. It should be remarked that the formulation can be transformed to a mixed-integer linear formulation. Initial on/off states of the generators are $u_{i 0}=u_{i, \text { init }}, i \in \mathcal{I}_{j}, j \in \mathcal{J}$.

Indirect costs include opportunity costs of releasing water, as the water could be stored and saved for future generation. Including these opportunity costs avoids end effects such as the tendency of the problem solution to empty the reservoir in the final stage. Such costs are measured as the future value of stored water, represented by the functions $V_{j}(),. j \in \mathcal{J}$, and available from more long-term models.

These functions are concave with respect to the stored water and approximated by a set of piecewise linear functions to support a linear formulation. The opportunity costs are measured by:

$$
\sum_{j \in \mathcal{J}}\left(V_{j}\left(l_{j 0}\right)-V_{j}\left(l_{j T}\right)\right)
$$

where

$$
V_{j}\left(l_{j t}\right)=\min _{h \in \mathcal{H}}\left\{d_{h j}^{1} l_{j t}+d_{h j}^{2}\right\}, \quad j \in \mathcal{J}, \quad t \in \mathcal{T}
$$

and where $d_{h j}^{1}, d_{h j}^{2}, h \in \mathcal{H}, j \in \mathcal{J}$ are the coefficients of the concave water value functions (Figure 4), and $\mathcal{H}=\{1, \ldots, H\}$, where $H$ is the number of piecewise linear functions used. Again, the formulation corresponds to a mixed-integer linear formulation. 


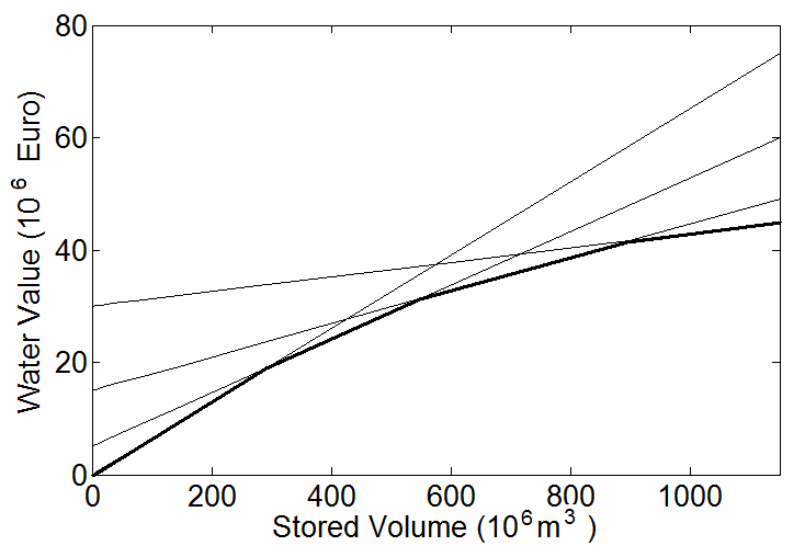

Figure 4 - Water value functions of reservoir Holen 12

To impose limits on the power generation the following constraints are included:

$$
u_{i t} w_{i}^{\min } \leq w_{i t} \leq u_{i t} w_{i}^{\max }, \quad i \in \mathcal{I}_{j}, \quad j \in \mathcal{J}, \quad t \in \mathcal{T}
$$

where $w_{i}^{\min }$ and $w_{i}^{\max }$ are the minimum and maximum generation level of each unit $i$. Similar bounds apply to the discharges, i.e.,

$$
u_{i t} v_{i}^{\min } \leq v_{i t} \leq u_{i t} v_{i}^{\max }, \quad i \in \mathcal{I}_{j}, \quad j \in \mathcal{J}, t \in \mathcal{T}
$$

where $v_{i}^{\min }$ and $v_{i}^{\max }$ are the minimum and maximum discharge levels of each unit $i$. The following constraints are also included to impose a limit to the total discharge of the turbines of each plant:

$$
\sum_{i \in \mathcal{I}_{j}} v_{i t} \leq V_{j}^{\max }, j \in \mathcal{J}, t \in \mathcal{T}
$$

where $V_{j}^{\max }, j \in \mathcal{J}$ is the maximum discharge from each set of turbines of each reservoir.

To impose limits on storage level the following constraints are included:

$$
l_{j}^{\min } \leq l_{j t} \leq l_{j}^{\max }, \quad j \in \mathcal{J}, t \in \mathcal{T}
$$

where $l_{j}^{\min }, j \in \mathcal{J}$ and $l_{j}^{\max }, j \in \mathcal{J}$ denote minimal and maximal storage levels.

The water balance must be imposed to each reservoir at each hour, such that the final storage from the previous period plus the inflow equals the final storage minus the discharged and spilled water. In the case of the upper reservoirs the balance equations are

$$
l_{j t}-l_{j, t-1}+v_{j t}+r_{j t}=\nu_{j t}, \quad t \in \mathcal{T}, \quad j \in \mathcal{J} \backslash\{3\}
$$

where $\nu_{j t}, j \in \mathcal{J}, t \in \mathcal{T}$ are the inflows from upstream. In the case of the lower reservoir $(j=3)$, the balance equations are:

$$
l_{3 t}-l_{3, t-1}+v_{3 t}+r_{3 t}=\nu_{3 t}+\sum_{j=1}^{2}\left(v_{j, t-\tau_{j}}+r_{j, t-\tau_{j}}\right), \quad t \in \mathcal{T}
$$

where $\tau_{j}$ is the time delay between each upper reservoir $j \in \mathcal{J} \backslash\{3\}$ and the lower reservoir $(j=3)$. The initial storage levels are

$$
l_{j 0}=l_{j, \text { init }}, \quad j \in \mathcal{J}
$$


The generation level is a function of the water discharge from the reservoir and the net water head of the power station. The net water head is the difference between the headwater elevation and the tailwater elevation and whereas the former is a function of the reservoir storage level, the latter is a function of the discharge. It is, however, assumed that the net water head only varies with the discharge over the course of the short-term planning horizon. The assumption is justified in the case of relatively small storage level variations, which holds for the case study. Ignoring some head variation effects, the relation between generation and discharge is approximated fairly well by a concave function, and is approximated by a set of piecewise linear functions to support a linear formulation. Hence,

$$
w_{i t}=G_{i}\left(v_{i t}\right), \quad i \in \mathcal{I}_{j}, \quad j \in \mathcal{J}, \quad t \in \mathcal{T}
$$

where

$$
G_{i}\left(v_{i t}\right)=\min _{k \in \mathcal{K}}\left\{f_{k i}^{1} v_{i t}+f_{k i}^{2}\right\}, \quad i \in \mathcal{I}_{j}, \quad j \in \mathcal{J}, \quad t \in \mathcal{T}
$$

and the coefficients of the piecewise linear functions are $f_{k i}^{1}, f_{k i}^{2}$, $k \in \mathcal{K}, \quad i \in \mathcal{I}_{j}, \quad j \in \mathcal{J}$ (Figure 5), and $\mathcal{K}=\{1, \ldots, K\}$, where $K$ is the number of functions used.



Figure 5 - Power generation functions of the third turbine of Brokke

Since we specify the piecewise functions for both power generation and water values exogenously, it is a relatively important to note that the model chooses to operate close to the intersections of these curves. It means that we should be very careful in specifying these curves.

To define the amount of energy that should be generated in each hour the set of unit commitment constraints must be included to determine that the total generation must be equal the sum of the accepted bids in the day-ahead market and the adjustments made by the trades in the Elbas market:

$$
y_{t}+\sum_{b \in \mathcal{B}: t \in b} y_{b}+z_{t}=\sum_{i \in \mathcal{I}_{j}} w_{i t}, \quad j \in \mathcal{J}, \quad t=1, \ldots, T
$$




\section{THE STOCHASTIC PROGRAMMING MODEL}

Stochastic programming is widely applied to solve problem with uncertainties. For an introduction to the subject, see Birge and Louveaux (1997), or Kall and Wallace (1994). A stochastic program is characterized by decisions taken according to information flow, and is divided into stages.

For hydropower production, data uncertainty can arise with respect to reservoir inflows and market prices. However, since the idea is to analyze uncertainty that relates directly to bidding, and due to the time horizon considered ( 24 hours), inflows are assumed to be deterministic and only prices are stochastic.

After the day-ahead prices are set, there is still uncertainty concerning the development of the Elbas prices. As information is revealed gradually, a multistage formulation is suitable. However, we approximate the problem using two stages, collapsing the dynamic prices and trading opportunities into only one opportunity for trading in Elbas for each delivery hour in the second stage. So there will be one Elbas price and one corresponding Elbas trading opportunity $z_{t}$ for each hour $t \in \mathcal{T}$.

The first stage concerns the decisions before observing uncertainty and involves the bidding process, whereas the second stage includes the trading decisions in the Elbas market and real-time hydropower production. As bids are submitted before the market has cleared, prices are unknown at the time of first-stage decision making. In contrast, second-stage decision making is put off until the market has cleared and takes advantage of the additional information from observing the spot and Elbas prices. The aim is to obtain the optimal bidding strategies in terms of expected sales and production profit.

To incorporate uncertainty, market spot prices $\left\{\rho_{t}\right\}_{t \in \mathcal{T}}$ are assumed to be described as a stochastic process whose distribution is known and given by a finite number of realizations $\mathcal{S}=\{1, \ldots, S\}$, referred to as scenarios. Denote the scenario probabilities by $\pi^{s}, s \in \mathcal{S}$ and the corresponding market prices by

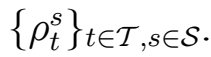

To incorporate the uncertainty in the Elbas prices we must take into account that there is a high correlation between spot prices and the average Elbas prices (equal to 0.80 using the historical data from 1 January to 30 November 2007).

Given that Norway was not participating in the Elbas market when this analysis was made, the correlation mentioned above was calculated using spot prices from Sweden, which is, among the Elbas participants, the biggest country in volume traded. It is important to note that the trading system automatically controls the cross-border capacity between the areas of Nord Pool. If there is no capacity for energy transmission between two given areas, two agents, one in each area, are not allowed to trade energy. Therefore the Elbas prices observed by each agent are correlated to the spot prices in their own area.

The historical series of the logarithm of the Elspot prices in Sweden (black line) and the logarithm of the average Elbas prices (gray line) are plotted in Figure 6. Figure 7 shows the regression between them. 


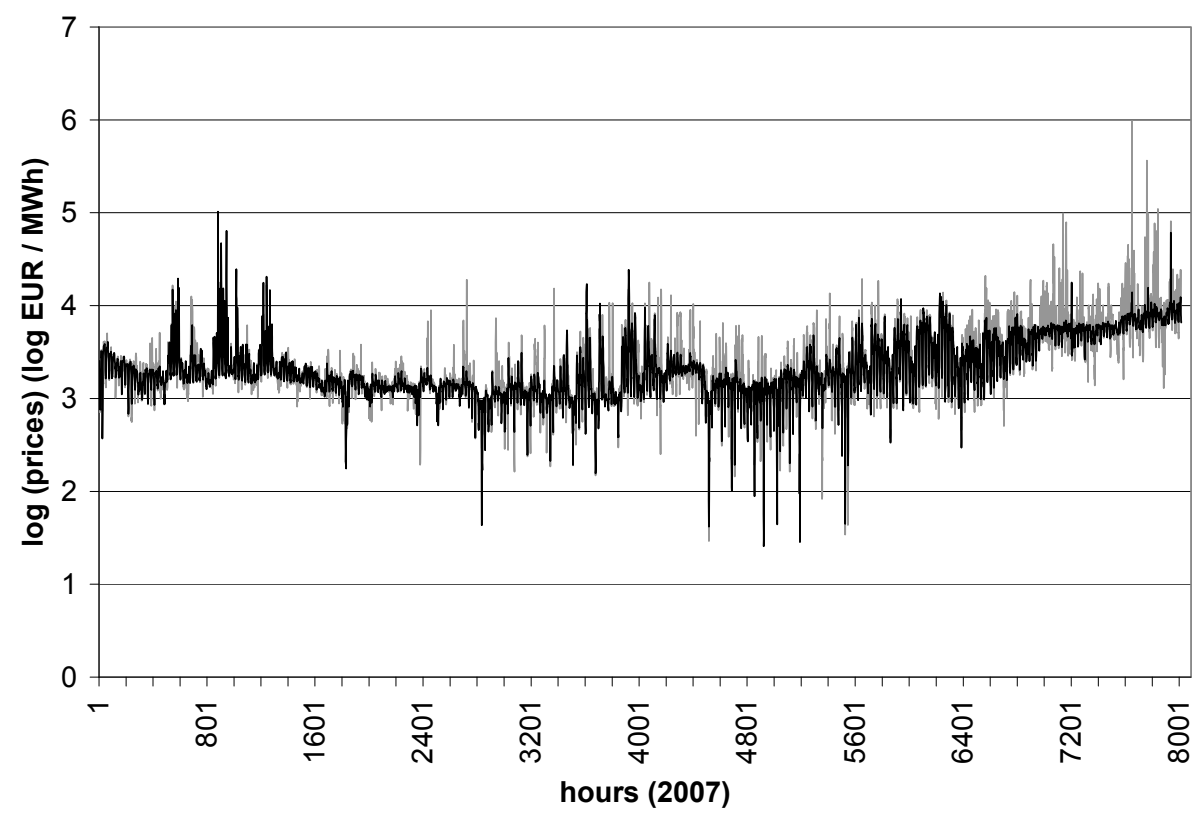

Figure 6 - Historical series of the logarithm of the spot prices in Sweden (black line) and logarithm of the Elbas prices (gray line) - (1 January to 30 November 2007)

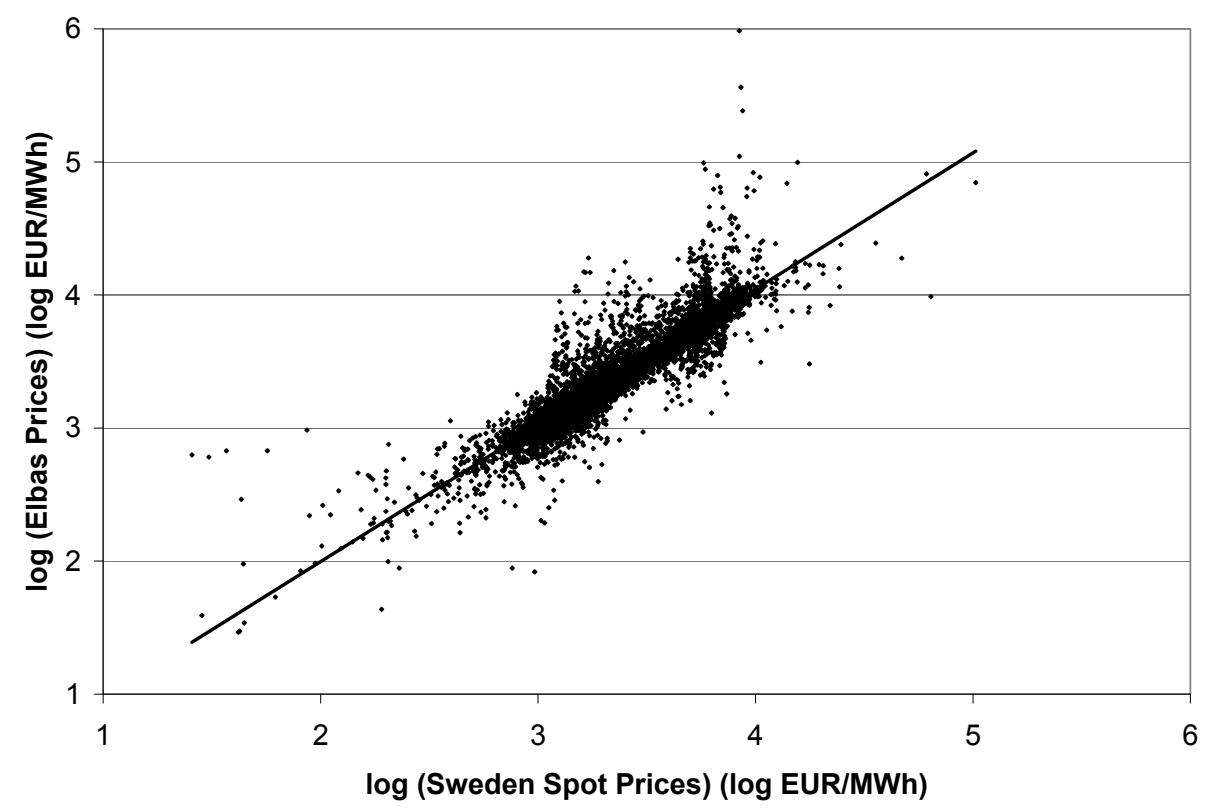

Figure 7 - Regression between the logarithm of the spot prices and the logarithm of the Elbas prices (2007)

Because of the high correlation, we assume that the Elbas prices are described by a stochastic process whose distribution is conditioned on the spot prices of each area. Thus, for each spot prices scenario $s \in \mathcal{S}$ we generate a finite number of conditional realizations of Elbas prices $e \in \mathcal{E}$, where $\mathcal{E}=\{1, \ldots, E\}^{4}$. Denote the scenario probabilities of Elbas prices by $\pi^{e}, e \in \mathcal{E}$ and the corresponding Elbas

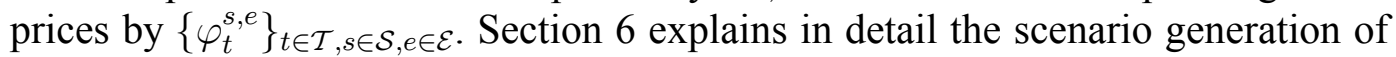

\footnotetext{
${ }^{4} \mathrm{We}$ assume that there is always the same number of scenarios of Elbas $(E)$ for each scenario of spot price $s$.
} 
spot and Elbas prices used in this work.

Whereas first-stage decisions $x_{i t}, x_{i b}, i \in \mathcal{I}, t \in \mathcal{T}, b \in \mathcal{B}$, i.e., volumes bid, are independent of future market prices, second-stage decisions $z_{t}^{s, e} \in \mathbb{R}, v_{i t}^{s, e}, w_{i t}^{s, e}$, $r_{j t}^{s, e}, l_{j t}^{s, e} \in \mathbb{R}_{+}, u_{i t}^{s, e} \in\{0,1\}$, i.e., volumes traded on Elbas and production decisions, are dependent on realizations of future market prices and are indexed by the spot and Elbas price scenarios superscripts $s \in \mathcal{S}, e \in \mathcal{E}$. Note that the volumes dispatched, $y_{t}^{s}$ and $y_{b}^{s}$, do not depend on the Elbas prices scenarios, but only on the realizations of future spot prices, and are indexed by only the spot price scenarios superscripts $s \in \mathcal{S}$.

The system operators require Elspot bids to be unbiased. This means that a producer must not expect to generate more or less than what is indicated by his Elspot bids ${ }^{5}$. In our case, it implies that Elbas trading is only used for unexpected situations, and translates into the following constraint:

$$
\sum_{s \in \mathcal{S}} \pi^{s}\left(\sum_{e \in \mathcal{E}} \pi^{e} z_{t}^{s, e}\right)=0, \quad t=1, \ldots, T
$$

Figure 8 shows the scheme of the decision process for a simple case with 3 spot prices scenarios, and each of them with 3 Elbas prices scenarios. The black circles represent decisions made by the producer, whereas the white ones correspond to variables calculated after the $1^{\text {st }}$ stage decisions.

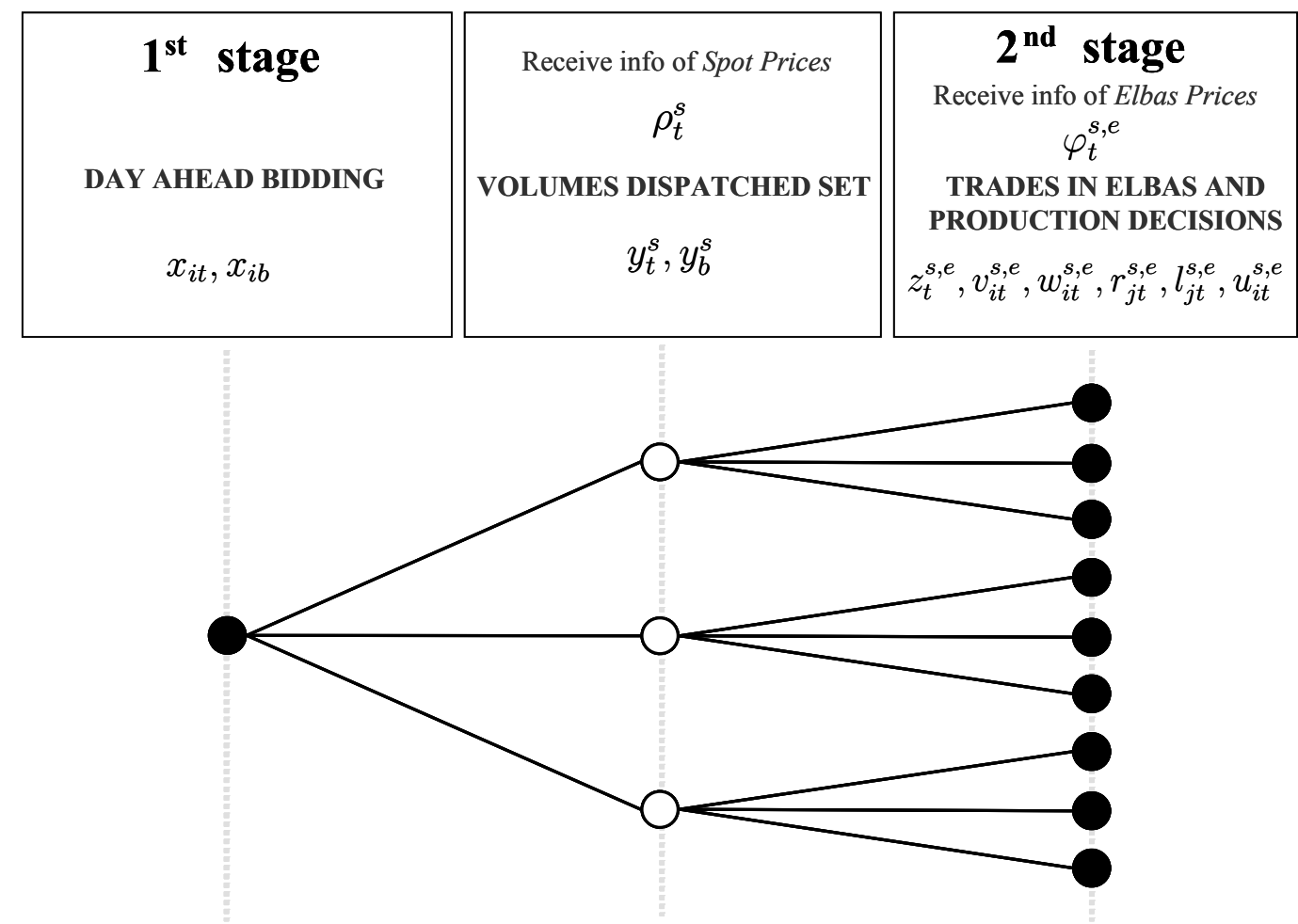

Figure 8 - Decision process scheme

The stochastic program consists of maximizing the expected sales and production profit subject to the bidding and operational constraints. The constraints (3)-(5) couples first-stage and second-stage decisions through the relation between

\footnotetext{
${ }^{5}$ This is because the system operators want the Elspot market to be the de facto spot market for physical delivery of power; they do not want shorter term markets like Elbas to assume this role.
} 
volumes bid and volumes dispatched whereas the constraints (7)-(21) applies to second-stage decisions only and model hydropower production. The two-stage stochastic mixed-integer program formulated as a deterministic equivalent is

$$
\begin{aligned}
& \max \sum_{s \in \mathcal{S}} \pi^{s}\left(\sum_{t \in \mathcal{T}} \rho_{t}^{s} y_{t}^{s}+\sum_{b \in \mathcal{B}} \bar{\rho}_{b}^{s} y_{b}^{s}\right. \\
& \left.+\sum_{e \in \mathcal{E}} \pi^{e}\left(-\sum_{j \in \mathcal{J}}\left(V_{j}\left(l_{j 0}\right)-V_{j}\left(l_{j T}^{s, e}\right)\right)-\sum_{t \in \mathcal{T}} \sum_{j \in \mathcal{J}} \sum_{i \in \mathcal{I}_{j}} S_{i}\left(u_{i, t-1}^{s, e}, u_{i t}^{s, e}\right)+\sum_{t \in \mathcal{T}} \varphi_{t}^{s, e} z_{t}^{s, e}\right)\right) \\
& \text { s.t. } \quad(3),(5),(8),(10),(11)-(21), \\
& x_{i t}, x_{i b} \in \mathbb{R}_{+}, i \in \mathcal{I}, t \in \mathcal{T}, b \in \mathcal{B} \\
& y_{t}^{s}, y_{b}^{s}, v_{i t}^{s, e}, w_{i t}^{s, e}, r_{j t}^{s, e}, l_{j t}^{s, e} \in \mathbb{R}_{+}, z_{t}^{s, e} \in \mathbb{R}, u_{i t}^{s, e} \in\{0,1\}, \\
& i \in \mathcal{I}_{j}, j \in \mathcal{J}, t \in \mathcal{T}, b \in \mathcal{B}, s \in \mathcal{S}, e \in \mathcal{E}
\end{aligned}
$$

Note that the decisions made on stage 1 (day-ahead hourly bids $x_{i t}$ and block bids $x_{i b}$ ) are the same for all realizations of spot and Elbas prices, and only constraints (3) and (5), which represent the accepted hourly bids and accepted block bids, are binding the scenarios together. All the other constraints are related to the physical production and depend on each price scenario.

Equations (3) and (5) can be simplified if the price points $i \in \mathcal{I}$ are fixed in advance. For a fixed $t \in \mathcal{T}$ and $s \in \mathcal{S}$, the market price $\rho_{t}^{s}$ is located between two adjacent points, and the remaining price points are irrelevant for determining the volume to be dispatched. Letting $i(t, s)=\max \left\{i \in \mathcal{I}: p_{i} \leq \rho_{t}^{s}\right\}$, the accepted bid $\left(y_{t}^{s}, \rho_{t}^{s}\right)$ is located on the line segment between $\left(x_{i(t, s) t}, p_{i(t, s)}\right)$ and $\left(x_{i(t, s)+1 t}, p_{i(t, s)+1}\right)$. From this, (3) is equivalent to

$y_{t}^{s}=\frac{\rho_{t}^{s}-p_{i(t, s)}}{p_{i(t, s)+1}-p_{i(t, s)}} x_{i(t, s)+1 t}+\frac{p_{i(t, s)+1}-\rho_{t}^{s}}{p_{i(t, s)+1}-p_{i(t, s)}} x_{i(t, s) t}, \quad t \in \mathcal{T}, s \in \mathcal{S}$

Similarly, letting $i(b, s)=\max \left\{i \in \mathcal{I}: p_{i} \leq \bar{\rho}_{b}^{s}\right\}$, (5) is equivalent to

$$
y_{b}^{s}=\sum_{j \leq i(b, s)} x_{j b}, \quad b \in \mathcal{B}, s \in \mathcal{S}
$$

The price points $p_{i}, i \in \mathcal{I}$ are fixed in advance within the interval of spot prices realizations, and are equidistant to each other.

\section{SCENARIO GENERATION}

Some of the widely applied methods in price forecasting and simulation are based on time series analysis, by modeling an advanced stochastic process calibrated from historical price profiles. The stochastic process of hourly day-ahead prices constitutes a time series characterized by seasonal changes, periodic cycles and stochastic variations. Basically, ARMA processes are a specific class of stochastic processes adopted for the analysis of time series and date back to Box and Jenkins (1970). The use of ARMA models and variants of these to forecast hourly dayahead prices are often seen in the literature, see Contreras et al. (2003), Nogales et al.(2002) and Haldrup and Nielsen (2006).

However, the electricity prices are traditionally assumed to be driven by models 
whose error terms follow a normal distribution with constant variance. Unfortunately, these models neglect their changeable volatility, and only a few studies on price forecasting pay attention to the modeling of the dynamic properties of the price variance. This paper, following Garcia and Contreras (2005), focuses on day-ahead price simulation using a GARCH approach. The Autoregressive Conditional Heteroskedastic (ARCH) class of models was introduced by Engle (1982), and the extended ARCH model called GARCH (Generalized Autoregressive Conditional Heteroskedastic) was proposed by Bollerslev (1986). GARCH models assume the conditional variance of the error term to be serially correlated and, therefore, we do not have to assume that it has zero mean and constant variance, as with an ARMA model.

Two kinds of scenario generation models are designed in this work, one for simulating day-ahead prices and another for simulating Elbas prices. Both models are based on GARCH. The day-ahead spot prices are modeled as an ARMA process with GARCH error components, whereas the Elbas prices are modeled as an ARMAX process with GARCH error components and spot prices as explanatory variables. The next two subsections explain the two models in detail.

\subsection{Scenario generation for day-ahead prices}

The first model is designed for scenario generation of spot prices for Norway, more specifically for the NO1 area, where the set of hydropower plants from the upper part of the Otra cascade is located.

The data set used consists of hourly spot prices in the NO1 region from 1 January 2007 to 30 November 2007, and was obtained from Nord Pool. The model consists of an ARMA process with GARCH error components.

The general $\operatorname{ARMA}(\mathrm{p}, \mathrm{q})$ process is formulated as

$$
y_{t}=C+\sum_{i=1}^{p} \phi_{i} y_{t-i}+\sum_{j=1}^{q} \theta_{j} \varepsilon_{t-j}+\varepsilon_{t}
$$

or,

$$
\Phi(B) y_{t}=C+\Theta(B) \varepsilon_{t}, t \in \mathbb{Z}
$$

where $\Phi(B)$ and $\Theta(B)$ are polynomials of the form $\Phi(B)=1-\sum_{i=1}^{p} \phi_{i} B^{i}$ and $\Theta(B)=1+\sum_{j=1}^{q} \theta_{j} B^{j}$ and $B$ denotes the back-shift operator, i.e., $B^{i} y_{t}=y_{t-i}$.

The innovations $\left\{\varepsilon_{t}\right\}_{t \in \mathbb{Z}}$ are assumed a Gaussian white noise process, i.e., $\varepsilon_{t}, t \in \mathbb{Z}$ are independent normally distributed random variables with zero mean and constant variance, i.e., $\mathbb{E}\left(\varepsilon_{t}\right)=0$ and $\mathbb{E}\left(\varepsilon_{t}^{2}\right)=\sigma^{2}$, for all $\mathrm{t}$; and $\mathbb{E}\left(\varepsilon_{t} \varepsilon_{s}\right)=0$, for $s \neq t$.

The constant variance assumption $\mathbb{E}\left(\varepsilon_{t}^{2}\right)=\sigma^{2}$ does not necessarily need to hold, and a generalized heteroscedastic error specification is strongly supported by the data, which significantly improves the model's goodness of fit.

The general GARCH(p,q) model for the conditional variance of innovations, 
$\sigma_{t}^{2}=\operatorname{Var}\left(\varepsilon_{t} \mid \psi_{t-1}\right)$, where $\psi_{t-1}$ is the set of all information available at time $t-1$, is formulated as:

$$
\begin{aligned}
& \varepsilon_{t}=z_{t} \sigma_{t}, \text { where } z_{t} \sim \operatorname{iid} N(0,1) \\
& \sigma_{t}^{2}=K+\sum_{i=1}^{p} \alpha_{i} \sigma_{t-i}^{2}+\sum_{j=1}^{q} \beta_{j} \varepsilon_{t-j}^{2}
\end{aligned}
$$

The GARCH model considers the conditional variance as time dependent, i.e., the dynamics of $\varepsilon_{t}^{2}$ can be explained through past volatility shocks $\varepsilon_{t-i}^{2}$. Note that $\frac{\varepsilon_{t}}{\sigma_{t}}$, which is equal $z_{t}$, is a white noise with zero mean and variance equal to 1 , and this assumption is tested on the validation of the fitted model (step 3 below).

The development of the proposed model follows the steps:

1. Identify a statistical model of the historical data

2. Estimate the parameters of the model

3. Validate the model

4. Use simulation to generate scenarios

1. For identification we use historical data of hourly spot prices, and the analysis begins with a careful inspection of the main characteristics of the time series. Non-constant mean and variance as well as calendar effects and seasonal trends corresponding to daily and weekly periodicity are observed. In the creation of a trial model the data is made stationary by a transformation of the original data. The price series is converted to a return series (first difference of the logarithm of the prices), i.e., $y_{t}=\log \frac{\rho_{t+1}}{\rho_{t}}=\log \rho_{t+1}-\log \rho_{t}$, which results in a more stable variance and avoids negative numbers for generated price scenarios ${ }^{6}$. The structure of the polynomials is determined by investigating the autocorrelations and partial autocorrelations of the transformed data. In successive trials using the general ARMA-GARCH model (25)-(27)-(28), refinements can be made based on the autocorrelations and partial autocorrelations of the residuals, and the final model identified is:

$$
\begin{aligned}
& \left(1-\phi_{1} B^{1}-\phi_{2} B^{2}-\phi_{4} B^{4}-\phi_{23} B^{23}-\phi_{24} B^{24}-\phi_{48} B^{48}-\phi_{72} B^{72}\right. \\
& \left.-\phi_{120} B^{120}-\phi_{144} B^{144}-\phi_{167} B^{167}-\phi_{168} B^{168}-\phi_{336} B^{336}-\phi_{504} B^{504}\right) \\
& \times(1-B) \log \rho_{t}=C+\varepsilon_{t}
\end{aligned}
$$

where $\varepsilon_{t}$ is an GARCH(25) model, such that (27)-(28) are:

$$
\varepsilon_{t}=z_{t} \sigma_{t}, \text { where } z_{t} \sim \operatorname{iid} N(0,1)
$$

\footnotetext{
${ }^{6}$ The correlation between the return series of spot prices and the return series of Elbas prices is 0.54 for the historical data used in this work.
} 


$$
\sigma_{t}^{2}=K+\sum_{i=1}^{4} \alpha_{i} \sigma_{t-i}^{2}+\sum_{j=22}^{25} \alpha_{j} \sigma_{t-j}^{2}
$$

2. On completion of the identification parameter estimates may be computed by the use of maximum likelihood optimization. Estimates are displayed in Table 1.

3. The model is validated by testing the assumptions of the ARMA-GARCH model proposed in step 1. The assumption of a Gaussian white noise process made on the standardized innovations (fitted innovations ${ }^{7}$ divided by conditional standard deviations) is tested by studying their autocorrelation and partial autocorrelation as well as the Ljung-Box statistics (test for no correlation, or randomness) and Engle's hypothesis test (test for presence of conditional volatility) for different lags. The AIC/BIC (Akaike and Bayesian information criteria) also helps to compare and penalize the excess of parameters of different models. When the statistical significance of the parameters and the residuals tests are validated the model is ready to be used for simulating. Otherwise, the model is refined in step 1 .

4. Simulation of price scenarios can be done by withdrawing the starting values $\rho_{t}, t=-504, \ldots, 0$ and $\varepsilon_{t}, t=-25, \ldots, 0$ from the historical data and sampling from the independent identically normally distributed random variables $z_{t}, t=1, \ldots, T$. Monte Carlo sampling has been used to simulate a large number of scenarios.

Steps 1-4 are all carried out using Matlab (GARCH Toolbox 2). We considered using reduction techniques on the generated scenarios (Heitsch and Römisch, 2006), however, we found the solutions stable enough using crude Monte Carlo sampling.

Table 1 - Parameter estimates of hourly day-ahead spot prices in the NO1 area

\begin{tabular}{c|ccccccc}
\hline Parameter & $C$ & $\phi_{1}$ & $\phi_{2}$ & $\phi_{4}$ & $\phi_{23}$ & $\phi_{24}$ & $\phi_{48}$ \\
Estimates & $3.00 \mathrm{e}-04$ & 0.1821 & -0.0608 & -0.0462 & 0.0614 & 0.2125 & 0.0284 \\
\hline Parameter & $\phi_{72}$ & $\phi_{120}$ & $\phi_{144}$ & $\phi_{167}$ & $\phi_{168}$ & $\phi_{336}$ & $\phi_{504}$ \\
Estimates & 0.0492 & 0.0326 & 0.0480 & 0.0484 & 0.1466 & 0.0121 & 0.0354 \\
\hline Parameter & $K$ & $\alpha_{1}$ & $\alpha_{2}$ & $\alpha_{3}$ & $\alpha_{4}$ & $\alpha_{22}$ & $\alpha_{23}$ \\
Estimates & $4.44 \mathrm{e}-005$ & 0.3529 & 0.0737 & 0.0601 & 0.0138 & 0.0488 & 0.1898 \\
\hline Parameter & $\alpha_{24}$ & $\alpha_{25}$ & & & & & \\
Estimates & 0.1954 & 0.0655 & & & & & \\
\hline
\end{tabular}

For illustration purposes a number of demonstration scenarios are generated and plotted (Figure 9).

\footnotetext{
${ }^{7}$ Actual prices minus fitted values.
} 


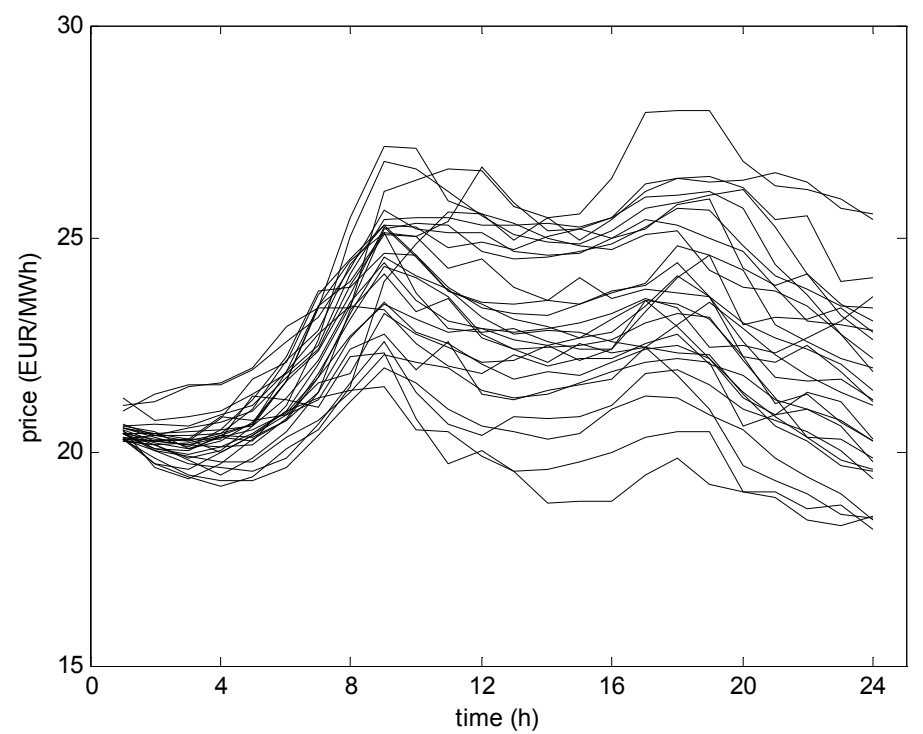

Figure $9-20$ simulation scenarios of spot prices in the NO1 area

\subsection{Scenario generation for Elbas prices}

The second model is designed for scenario generation for Elbas prices. As stated in Section 5, there is a high correlation between spot prices and the Elbas prices. Therefore, we assume that the Elbas prices are described by a stochastic process whose distribution is conditional on the spot prices, and the Elbas prices are modeled as an ARMAX process with GARCH error components and spot prices as explanatory variables.

Given that Norway had not entered Elbas when the study was carried out, we fit the ARMAX model using historical data of Elbas prices with spot prices from Sweden as explanatory variable. After that, to generate scenarios of Elbas prices to agents of the NO1 area, we use the fitted model and scenarios of spot prices in the NO1 area as explanatory variable. Here we assume that the relationship between Elbas prices and spot prices in Sweden is going to be similar to Elbas prices and spot prices in the NO1 area.

The data set used was obtained from Nord Pool and consists of hourly spot prices of Sweden and Elbas prices ${ }^{8}$ from 1 January 2007 to 30 November 2007.

The general ARMAX $(p, q)$ process is formulated as

$$
y_{t}=C+\sum_{i=1}^{p} \phi_{i} y_{t-i}+\sum_{j=1}^{q} \theta_{j} \varepsilon_{t-j}+\xi X_{t}+\varepsilon_{t}
$$

where the innovations $\left\{\varepsilon_{t}\right\}_{t \in \mathbb{Z}}$ are assumed a Gaussian white noise process, $X_{t}$ is the explanatory variable and $\xi$ is the regression coefficient.

Again, assuming the general $\operatorname{GARCH}(\mathrm{p}, \mathrm{q})$ model for the conditional variance of the innovations, $\sigma_{t}^{2}=\operatorname{Var}\left(\varepsilon_{t} \mid \psi_{t-1}\right)$ is formulated as in (27)-(28), where $\psi_{t-1}$ is the set of all information available at time $t-1$.

\footnotetext{
${ }^{8}$ Average of the prices in Elbas during each hour.
} 
The design of the proposed GARCH model for Elbas prices follows the same 3 first steps presented in the Subsection 6.1. Again, in order to get a more stable variance and avoid negative numbers for generated scenarios, the Elbas prices series is also converted to a return series. The difference from Subsection 6.1 is the simulation part (step 4), which considers the spot prices realizations in the $\mathrm{NO} 1$ area as explanatory variables.

In successive trials using the general GARCH model, refinements can be made based on the autocorrelations and partial autocorrelations of the residuals, and the final model is identified as:

$$
\begin{aligned}
& \left(1-\phi_{1} B^{1}-\phi_{4} B^{4}\right) \\
& \times(1-B) \log \varphi_{t}=C+\xi_{h(t)} X_{t}+\varepsilon_{t}
\end{aligned}
$$

where $X_{t}$ is the explanatory variable, the return series of the logarithm of the spot prices, and $\varepsilon_{t}$ is a $\operatorname{GARCH}(24,4)$ model, such that $(27)-(28)$ are:

$$
\begin{aligned}
& \varepsilon_{t}=z_{t} \sigma_{t}, \text { where } z_{t} \sim \text { iid } N(0,1) \\
& \sigma_{t}^{2}=K+\sum_{i=23}^{24} \alpha_{i} \sigma_{t-i}^{2}+\sum_{j=1}^{4} \beta_{j} \varepsilon_{t-j}^{2}
\end{aligned}
$$

$\xi$ 's are the regression coefficients indexed by $h(t)$, a function whose output is one of the 24 hours of the day corresponding to period $t$. For example, $h(1)=1$, $h(2)=2, \ldots, h(24)=24, h(25)=1, h(26)=2, \ldots, h(48)=24$, and so on. Therefore, $\xi_{k}, k=$ $\{1, \ldots, 24\}$, are the coefficients estimated for each of the 24 hours of the day ${ }^{9}$. Estimates of all parameters are displayed in Table 2.

Table 2 - Parameter estimates of hourly day-ahead spot prices in the NO1 area

\begin{tabular}{l|ccccccc}
\hline Parameter & $C$ & $\phi_{1}$ & $\phi_{4}$ & $\xi_{1}$ & $\xi_{2}$ & $\xi_{3}$ & $\xi_{4}$ \\
Estimates & -0.0814 & 0.5653 & 0.0206 & 0.4221 & 0.4200 & 0.4232 & 0.4274 \\
\hline Parameter & $\xi_{5}$ & $\xi_{6}$ & $\xi_{7}$ & $\xi_{8}$ & $\xi_{9}$ & $\xi_{10}$ & $\xi_{11}$ \\
Estimates & 0.4346 & 0.4516 & 0.4635 & 0.4720 & 0.4644 & 0.4479 & 0.4477 \\
\hline Parameter & $\xi_{12}$ & $\xi_{13}$ & $\xi_{14}$ & $\xi_{15}$ & $\xi_{16}$ & $\xi_{17}$ & $\xi_{18}$ \\
Estimates & 0.4529 & 0.4309 & 0.4332 & 0.4358 & 0.4310 & 0.4364 & 0.4451 \\
\hline Parameter & $\xi_{19}$ & $\xi_{20}$ & $\xi_{21}$ & $\xi_{22}$ & $\xi_{23}$ & $\xi_{24}$ & $K$ \\
Estimates & 0.44292 & 0.4421 & 0.44249 & 0.4280 & 0.4337 & 0.4196 & $3.67 \mathrm{e}-04$ \\
\hline Parameter & $\alpha_{23}$ & $\alpha_{24}$ & $\beta_{1}$ & $\beta_{2}$ & $\beta_{3}$ & $\beta_{4}$ & \\
Estimates & 0.2676 & 0.3624 & 0.2388 & 0.0941 & 0.0189 & 0.0182 & \\
\hline
\end{tabular}

9 As in the general ARMAX model (32), we could use only one coefficient $\xi$ for all hours, and in this case fewer parameters have to be estimated. This option was analyzed and the fitted model was compared to others using the AIC/BIC criteria, but the results are worse than using the more flexible model. 
For illustration purposes a number of demonstration scenarios have been generated. Figure 10 shows 10 Elbas scenarios generated for 3 different spot prices in the NO1 area (thick curves).


Figure 10 - 10 Elbas scenarios generated for 3 different spot price scenarios in the NO1 area

\section{CASE STUDY}

We consider three hydroelectric stations with total installed capacity of $719 \mathrm{MW}$ owned by Agder Energi, a Norwegian producer. To generate scenarios of dayahead prices and Elbas prices Nord Pool has provided real data from 2007 of the NO1 area in Norway, Sweden and Elbas market.

Three kinds of scenario sets are used in the case study: 20, 40 and 60 realizations of day-ahead spot prices for the first-stage, with 10 scenarios of Elbas prices to each scenario of spot price. Basically the sets correspond to a total of 200, 400 and 600 scenarios in the second-stage.

The problem is a large-scale mixed-integer linear program and is solved with Xpress-MP v2008 on an Intel Core 2 Duo $1.60 \mathrm{GHz}$ processor with 2 GB RAM. Table 3 shows the results summary of problem sizes and computational times for each scenario set. 
Table 3 - Summary of problem sizes and computational times

\begin{tabular}{c|ccc}
\hline & \multicolumn{3}{|c}{ \# Spot price scenarios } \\
& 20 & 40 & 60 \\
\hline Linear variables & 136,369 & 271,989 & 407,609 \\
\hline Binary variables & 33,600 & 67,200 & 100,800 \\
\hline Constraints & 276,019 & 550,839 & 825,659 \\
\hline Computational time (s) & 87 & 191 & 349 \\
\hline
\end{tabular}

Figure 11 shows typical results of bidding curves for three given hours. In all cases of this example the generator aims to dispose its whole capacity (719 MW) for any spot price greater than $29 \mathrm{EUR} / \mathrm{MWh}$.

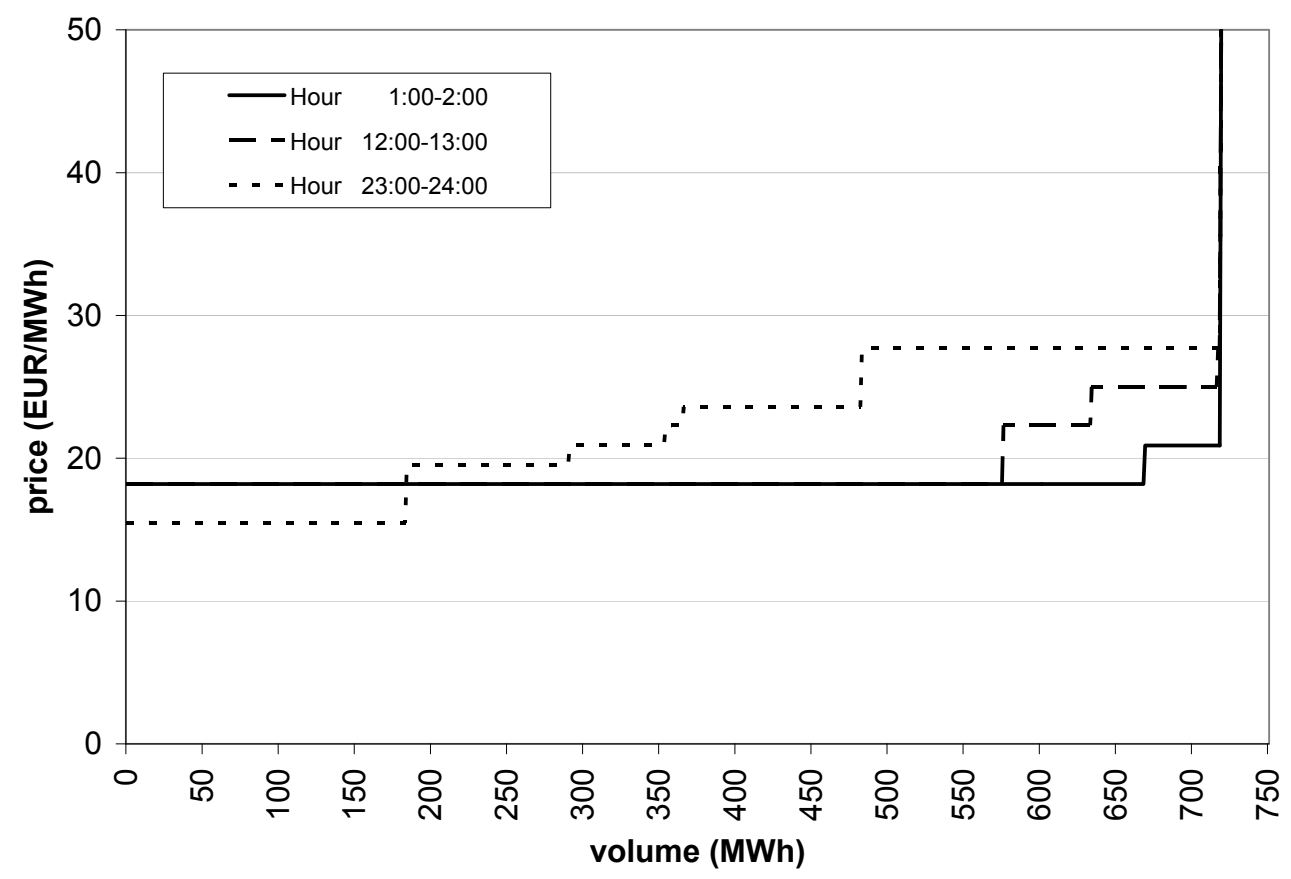

Figure 11 - Bidding curves for three given hours

The model stability is an important feature that we should pay attention to when evaluating the results (Kaut and Wallace, 2003a and 2003b). Basically we aim to guarantee that the errors' sampling applied to the scenario generation approach has an insignificant influence in the bidding decisions results. In other words, we run the same model using several scenarios generated with the same input and check if the optimal values of the objective function are reasonably similar to each other.

We carry out the stability tests by generating five times a set of 20, 40 and 60 spot prices scenarios in the NO1 area, with 10 Elbas prices scenarios for each of them. Table 4 shows the results. By using 20 scenarios of prices in the NO1 area the maximum deviation from the mean is $1 \%$, by using 40 the maximum deviation is 
$0.6 \%$ and by using 60 scenarios the maximum deviation is $0.3 \%$. For this reason, we conclude that the model results are stable, since the results of all scenario sets are sufficiently similar and never deviate more than $1 \%$ from the mean.

Table 4 - Stability Results: Optimal objective function for each scenario set and \% difference from the mean

\begin{tabular}{|c|c|c|}
\hline \multicolumn{3}{|c|}{$\begin{array}{l}20 \text { spot price scenarios in Norway } \\
\text { (with } 10 \text { Elbas price scenarios each) }\end{array}$} \\
\hline scenario set & Obj. function & $\%$ Diff. \\
\hline 1 & $1,085,478$ & $-0.7 \%$ \\
\hline 2 & $1,091,804$ & $-0.1 \%$ \\
\hline 3 & $1,098,273$ & $0.5 \%$ \\
\hline 4 & $1,104,355$ & $1.0 \%$ \\
\hline 5 & $1,083,559$ & $-0.9 \%$ \\
\hline mean & $1,092,694$ & \\
\hline \multicolumn{3}{|c|}{$\begin{array}{l}40 \text { spot price scenarios in Norway } \\
\text { (with } 10 \text { Elbas price scenarios each) }\end{array}$} \\
\hline scenario set & Obj. function & $\%$ Diff. \\
\hline 1 & $1,091,496$ & $-0.5 \%$ \\
\hline 2 & $1,098,966$ & $0.2 \%$ \\
\hline 3 & $1,103,447$ & $0.6 \%$ \\
\hline 4 & $1,100,873$ & $0.3 \%$ \\
\hline 5 & $1,097,288$ & $0.0 \%$ \\
\hline mean & $1,098,414$ & \\
\hline \multicolumn{3}{|c|}{$\begin{array}{l}60 \text { spot price scenarios in Norway } \\
\text { (with } 10 \text { Elbas price scenarios each) }\end{array}$} \\
\hline scenario set & Obj. function & \% Diff. \\
\hline 1 & $1,090,202$ & $-0.3 \%$ \\
\hline 2 & $1,093,094$ & $0.0 \%$ \\
\hline 3 & $1,095,645$ & $0.2 \%$ \\
\hline 4 & $1,093,272$ & $0.0 \%$ \\
\hline 5 & $1,094,007$ & $0.1 \%$ \\
\hline mean & $1,093,244$ & \\
\hline
\end{tabular}

The main objective of this section is to measure the effect of considering the Elbas market on the day-ahead bidding decisions. The effect is measured by what we call value of considering Elbas, which is calculated by the percentage difference of two results:

(i) The optimal expected profit of the model considering Elbas trades, i.e., the deterministic equivalent defined in (22);

(ii) The optimal expected profit of the model (22) using fixed day-ahead bids calculated without considering Elbas trades.

Thus, the value of considering Elbas $\Delta(\%)$ is calculated by:

$$
\Delta(\%)=[(\mathrm{i})-(\mathrm{ii})] /(\mathrm{ii}) \times 100
$$

The whole process involves 3 steps:

1. Solve the complete model (22); 
2. Solve model (22) without considering Elbas (i.e., forcing the variables $z_{t}$ to equal 0 ) and save the day-ahead bidding solutions $x_{t}^{*}$ and $x_{b}^{*}$;

3. Solve the complete model (22) using the fixed day-ahead bidding solutions $x_{t}^{*}$ and $x_{b}^{*}$, but letting the variables $z_{t}$ be different from 0 .

Figures 12 and 13 show two examples of bidding curve resulting from model (22) considering and not considering Elbas. The dashed line is the bidding curve result of the model considering Elbas (step 1 above) and the full-drawn line is the bidding curve without considering Elbas (step 2 above). The differences of the curves results from the fact that in the case of considering Elbas the producer has more flexibility to change its generation in the future by trading energy in Elbas. Thus, for a given price, the volume output from model considering Elbas (dashed line) is occasionally lower than the output from model not considering Elbas (fulldrawn line), indicating that in the first case the generator decides to save some energy considering the possibility of trading energy in Elbas market within the next day.



Figure 12 - Bidding curves considering (dashed line) and not considering Elbas (full-drawn line) hour 23:00-24:00 




Figure 13 - Bidding curves considering (dashed line) and not considering Elbas (full-drawn line) hour 12:00-13:00

Table 5 shows the value of considering Elbas results for the first 3 sets of scenarios and 3 different Elbas trade liquidity bounds, which are specified by percentages of the total installed capacity of the plants: $5 \%, 10 \%$ and $20 \%$. The value of considering Elbas calculated are very low, never greater than $0.12 \%$. Furthermore, considering that the ARMA-GARCH model used for generating scenarios is very sensitive to the current volatility of spot and Elbas prices, the model is also tested with a different set of scenarios generated with initial conditions from a period with higher volatility. Table 6 shows the results and the higher volatility produced higher values of considering Elbas, but still low, never greater than $0.65 \%$.

Table 5 - Value of considering Elbas for different limits on Elbas trading volumes

\begin{tabular}{c|ccc}
\hline \multirow{2}{*}{$\begin{array}{c}\text { \# Spot price scenarios } \\
\text { (with 10 Elbas price scenarios each) }\end{array}$} \\
\hline $\begin{array}{c}\text { \% limit Elbas } \\
\text { trades }\end{array}$ & 20 & 40 & 60 \\
\hline $5 \%$ & $0.03 \%$ & $0.03 \%$ & $0.02 \%$ \\
\hline $10 \%$ & $0.05 \%$ & $0.06 \%$ & $0.05 \%$ \\
\hline $20 \%$ & $0.08 \%$ & $0.12 \%$ & $0.07 \%$ \\
\hline
\end{tabular}


Table 6 - Value of considering Elbas for different limits on Elbas trading volumes Different Initial conditions with higher volatility

\begin{tabular}{c|ccc}
\hline \multirow{2}{*}{} & \multicolumn{3}{|c}{ \# Spot price scenarios } \\
(with 10 Elbas price scenarios each) \\
\cline { 1 - 1 } $\begin{array}{c}\text { \% Limit Elbas } \\
\text { Trades }\end{array}$ & 20 & 40 & 60 \\
\hline $5 \%$ & $0.18 \%$ & $0.14 \%$ & $0.17 \%$ \\
\hline $10 \%$ & $0.35 \%$ & $0.25 \%$ & $0.35 \%$ \\
\hline $20 \%$ & $0.65 \%$ & $0.57 \%$ & $0.64 \%$ \\
\hline
\end{tabular}

\section{CONCLUSIONS}

This paper proposes a mixed-integer program to support the day-ahead bidding of a price-taker hydropower producer in Norway taking into account the possibility of trading energy in the Elbas market. The model corresponds to a stochastic programming problem with two stages: the one related to determining the bidding curve and the second to the Elbas trades and production decisions.

The uncertainty in day-ahead prices and Elbas prices are represented in the model by a set of scenarios generated with an ARMA-GARCH model calibrated from historical data.

The impact of considering the possibility of trading in the Elbas in the day-ahead bidding decision is measured, and for this we introduce the value of considering Elbas concept. The results indicate that, for a price-taker medium-sized producer, considering Elbas when bidding on the day-ahead market does not impact significantly its profit. Therefore, the day-ahead bidding problem can be modeled without Elbas trading, thus simplifying that operational challenge. Future work involves tests considering thermal producers, whose results may be different.

\section{ACKNOWLEDGEMENTS}

The authors would like to thank Klaus Vogstad at Agder Energi for indispensable information and Trine K. Kristoffersen of Technical University of Denmark for helpful discussions of the problem. We also thank Nord Pool for generously supplying access to price data. Faria appreciates financial support from $\mathrm{CNPq}$ (Conselho Nacional de Desenvolvimento Científico e Tecnológico) and CAPES (Coordenação de Aperfeiçoamento de Pessoal de Nível Superior). Fleten acknowledges support from the Research Council of Norway through project 178374/S30, and through the ELDEV project at Sør-Trøndelag University College. Finally we recognize the Norwegian research centre CenSES, Centre for Sustainable Energy Studies. 


\section{REFERENCES}

Anderson EJ, Philpott AB (2000) Optimal offer construction in electricity markets, Mathematics of Operations Research 27 (1), pp. 82-100

Birge JR, Louveaux F (1997) Introduction to Stochastic Programming, Springer, New York

Bollerslev T (1986), Generalized autoregressive conditional heteroscedasticity, Journal of Econometrics, vol. 31, pp. 307-327

Box GEP, Jenkins GM (1970) Time Series Analysis, Holden Day

Conjeno AJ, Nogales FJ, Arroyo JM (2002) Price-taker bidding strategy under price uncertainty, IEEE Transactions on Power Systems 17 (4), pp. 1081-1088

Contreras J, Espinola R, Nogales FJ, Conejo AJ (2003) ARIMA models to predict next-day electricity prices, IEEE Transactions on Power Systems 18 (3), pp. 1014-1019

Engle RF (1982) Autoregressive conditional heteroscedasticity with estimates of the variance of United Kingdom inflation, Econometrica, vol. 50, pp. 987-1007

Flatabø N, Haugstad A, Mo B, Fosso OB (2002), Short-term and medium-term generation scheduling in the Norwegian hydro system under a competitive power market structure, VIII SEPOPE'02, Brasil

Fleten S-E, Kristoffersen TK (2007) Stochastic programming for optimizing bidding strategies of a Nordic hydropower producer, European Journal of Operations Research 181, pp. 916-928

Fleten S-E, Kristoffersen TK (2008) Short-term hydropower production planning by stochastic programming, Computers \& Operations Research 35 (2008) 2656 - 2671

Fleten S-E, Pettersen E (2005) Constructing bidding curves for a price-taking retailer in the Norwegian electricity market, IEEE Transactions on Power Systems 20 (2), pp. 701-708

Fosso OB, Belsnes MM (2004) Short-term hydro scheduling in a liberalized power system, in: Proceedings of the International Conference on Power System Technology, 21-24, Singapore

Fosso OB, Gjelsvik A, Haugstad A, Mo BI, Wagensteen I (1999) Generation scheduling in a deregulated system. The Norwegian case, IEEE Transactions on Power Systems 14 (1), pp. 75-81

Garcia RC, Contreras J, Akkeren MV, Garcia JBC (2005) A GARCH forecasting model to predict day-ahead electricity prices, IEEE Transactions on Power Systems 20 (2), pp. 867-874

Haldrup N, Nielsen MO (2006) A regime switching long memory model for electricity prices, Journal of Econometrics 135 (1-2), pp. 349-376

Heitsch H, Römisch W (2009) Scenario tree reduction for multistage stochastic programs, Computational Management Science 6 (2), pp. 115-267.

Høyland K., Kaut M, Wallace SW (2003) A heuristic for moment-matching scenario generation, Computational Optimization and Applications 24, pp. 169-185

Kall P, Wallace W (1994) Stochastic Programming, Wiley, Chichester

Kaut M, Wallace SW (2003a) Stability analysis of a portfolio management model based on the conditional value-at-risk measure. Available in

http://work.michalkaut.net/CV_and_study/CVaR_stability_abstract.htm

Kaut M, Wallace SW (2003b) Evaluation of scenario-generation methods for stochastic programming. Available in

http://work.michalkaut.net/CV_and_study/SG_evaluation_abstract.htm

Kristoffersen TK, Fleten S-E (2009) The development of stochastic programming models for short-term power production and trading, in Bjørndal, Bjørndal and Rönnqvist (Eds) Energy, Natural Resource and Enviromental Economics, accepted for publication

Ladurantaye DD, Gerdreau M, Potvin JY (2007) Strategic Bidding for Price-Taker Hydroelectricity Producers, IEEE Transactions on Power Systems 22 (4), pp. 2187-2203 
Matlab. http://www.mathworks.com/

Nogales FJ, Conejo AJ, Espinola R (2002) Forecasting next-day electricity prices by time series models, IEEE Transactions on Power Systems 17 (2), pp. 342-349

Nordel Annual Report 2007. Available in http://www.nordel.org

Nowak MP, Nürnberg R, Römisch W, Schultz R, Westphalen M (2003) Stochastic programming for power production and trading under uncertainty. In: W. Jäger and H.J. Krebs, Editors, Mathematics - Key Technology for the Future, Springer, Berlin, pp. 623-636

Oliveira P, McKee S, Coles C (1993) Optimal scheduling of a hydro thermal power generation system, European Journal of Operations Research 71, pp. 334-340

Olsson M, Söder L (2008) Modeling Real-Time Balancing Power Market Prices Using Combined SARIMA and Markov Processes, IEEE Transactions on Power Systems 23 (2), pp. 443-450

Philpott AB, Craddock M, Waterer H (2000) Hydro-electric unit commitment subject to uncertain demand, European Journal of Operations Research 125, pp. 410-424

Shawwash ZK, Siu TK, Russel SO (2000) The B.C. Hydro short term hydro scheduling optimization model, IEEE Transactions on Power Systems 15 (3), pp. 1125-1131

Thénié J, Delft CV, Vial JP (2007) Automatic Formulation of Stochastic Programs Via an Algebraic Modeling Language, Computational Management Science 4, pp. 17-40.

Wallace SW, Fleten S-E (2003) Stochastic programming models in energy. In: A. Ruszczynski and A. Shapiro, Editors, Stochastic Programming, Handbooks in Operations Research and Management Science vol. 10, Elsevier, North-Holland, pp. 637-677

Wets RJ-B (1974) Stochastic Programs with Fixed Recourse: The Equivalent Deterministic Program, SIAM Review, vol. 16, no. 3, pp. 309-339

Xpress MP, http://www.dashoptimization.com/ 